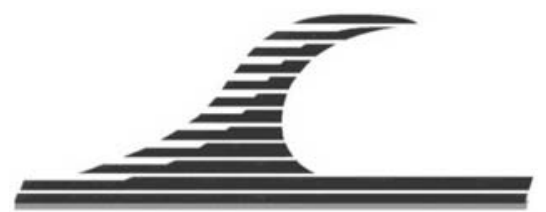

\title{
Trois siècles d'histoire hydro-sédimentaire et écologique du lac de Grand-Lieu, conséquences pour la gestion hydraulique
}

\author{
Eric MERCIER ${ }^{1}$, Martin SANCHEZ ${ }^{1}$, Pierre VACHER ${ }^{1}$
}

1. Université de Nantes, Faculté des Sciences et Techniques, UMR-6112 du CNRS - Planétologie et Géodynamique, 2, rue de la Houssinière, BP 92208, 44322 Nantes cedex 3, France.

Eric.Mercier@univ-nantes.fr, Martin.Sanchez@univ-nantes.fr ;

Pierre.Vacher@univ-nantes.fr

\section{Résumé :}

Dans un premier temps, les histoires hydraulique et sédimentaire du lac de Grand-Lieu, sont retracées depuis le début du XVIIIème siècle. Les évolutions mises en évidence, fortement induites par les activités humaines, se sont traduites, selon les époques, par des modifications écologiques positives ou négatives. Dans un second temps, nous montrerons que la très forte régression de la biodiversité du lac que l'on constate depuis le début des années 1960, s’inscrit dans son évolution hydraulique et sédimentaire. En conclusion nous proposerons des pistes pour une gestion, notamment hydraulique, plus en phase avec les nécessités écologiques.

\begin{abstract}
:
In the first part, the hydraulic and sedimentary history of Grand-Lieu Lake, are traced from the early eighteenth century. Some ecological changes, positive or negative according to the times, could be distinguished. These evolutions are strongly induced by human activities. In the second part, we show that the strong decline of the biodiversity observed since the early 1960s, is related to the hydraulic and sedimentary evolution. In conclusion, we propose ways for a hydraulic management of the lake more in line with ecological requirements.
\end{abstract}

Keywords: Shallow lake, Euthrophisation, Water management, Erosion, Turbidity.

Article issu d'une sélection des XIèmes Journées Nationales Génie Côtier Génie Civil qui se sont tenues aux Sables d'Olonne du 22 au 25 juin 2010. Accepté le 14 décembre 2010, en ligne le 14 octobre 2013.

Pour citer cet article :

MERCIER E., SANCHEZ M., VACHER P. (2013). Trois siècles d'histoire hydro-sédimentaire et écologique du Lac de Grand-Lieu, conséquences pour la gestion hydraulique. Revue Paralia, Vol. 6, pp 6.1-6.26. 


\section{Introduction}

Une proportion significative du territoire européen est concernée par des réglementations visant à la protection des écosystèmes et de la biodiversité ; par exemple, on recense $850000 \mathrm{~km}^{2}$ classés "Natura 2000", soit 18\% du territoire. Dans le détail, la réglementation peut varier fortement selon le degré de protection ou selon le pays. Dans tous les cas, la démarche de protection se traduit par des mesures de conservation qui visent à maintenir les caractéristiques des écosystèmes. Or, et c'est assez paradoxal, les écosystèmes ont "naturellement" tendance à évoluer et donc à perdre les spécificités qui ont justifié les mesures de protection. Cette évolution est classiquement considérée par les biologistes comme une "banalisation" et donc non souhaitable car contraire au maintien de la biodiversité (RAMADE, 1984). Généralement, la cinétique de cette évolution est suffisamment lente pour que les objectifs de conservation ne soient pas remis en cause à court terme. Mais parfois, l'évolution du milieu s'accélère ; cela concerne particulièrement les milieux humides et constitue souvent une réponse à la forte pression des facteurs abiotiques (modification du régime hydraulique, phénomènes sédimentaires ou érosifs rapides ...; SCHEFFER (1998), PIEGAY et al. (2003), DE MARSILY (2006), par exemple).

En cas d'évolution rapide, et pour préserver la spécificité originelle de l'écosystème, le gestionnaire doit abandonner son rôle de "conservateur" et intervenir dans la dynamique du milieu. Il est à noter que cette intervention, qui revient à lutter contre une évolution pouvant être perçue comme "naturelle", peut poser des problèmes de communication en direction du public et/ou de certains décideurs. Pour que l'intervention du gestionnaire soit efficace, il faut que les moteurs de l'évolution soient clairement identifiés et quantifiés. Souvent, en l'absence de recul temporel, le gestionnaire n’a d'autres solutions que d'envisager le problème à la lumière de modèles théoriques d'évolution trophique des milieux (par exemple : les lacs tendent à s'atterrir, les marais à évoluer vers les prairies, qui elles-mêmes ont tendance à se boiser etc..., RAMADE (1984)). Dans la plupart des cas, cette approche semble suffire. Mais ce n'est pas toujours le cas. Le but de cet article est de montrer, dans le cas de la zone humide du lac de Grand-Lieu, comment une analyse historique de l'évolution séculaire de ce milieu peut modifier totalement la vision que l'on avait de sa dynamique. Par voie de conséquence, cette analyse historique conduit à remettre en cause radicalement la politique de gestion actuelle.

\section{Le lac de Grand-Lieu}

\subsection{Les problèmes de conservation des écosystèmes}

Le lac de Grand-Lieu constitue le cœur d'une vaste zone humide (6000 ha) située à quelques kilomètres au sud-ouest de Nantes (44). Ce lac, et la zone humide qui 
Trois siècles d'histoire hydro-sédimentaire et écologique du lac de Grand-Lieu, conséquences pour la gestion hydraulique : 6.3

l'entoure (figure 1), constituent des milieux écologiques remarquables, et sont protégés par un arsenal de dispositifs juridiques européen (Natura 2000) et français (ZNIEFF Zone Naturelle d'Intérêt Ecologique, Faunistique et Floristique-, Site inscrit, Site classé, Réserve de chasse, Convention de Ramsar, Réserve Naturelle) (STRUILLOU et al., 2006). Actuellement deux organismes principaux se partagent la gestion du lac: la Société Nationale de Protection de la Nature (SNPN), pour la partie centrale et occidentale, et la Fondation Nationale pour la Protection des Habitats de la Faune Sauvage (Wildelife Habitats Foundation, WHF) pour la partie orientale. La maîtrise des niveaux d'eaux reste néanmoins de la compétence de l'Etat par l'intermédiaire d'un règlement d'eau défini par un arrêté ministériel (PIERRELEE \& LAMPRIER, 2004).

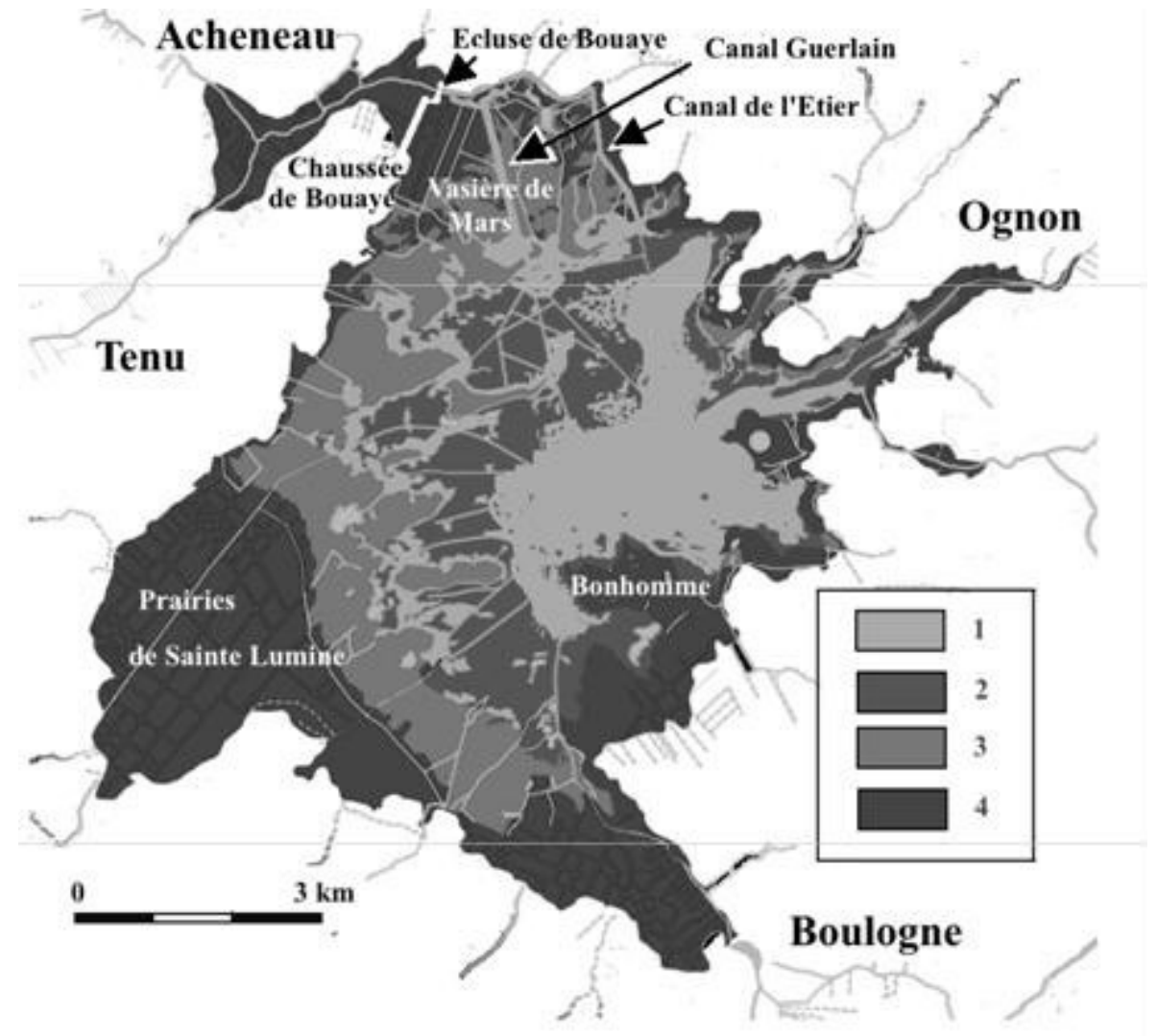

Figure 1. Les principaux milieux et le contexte hydraulique du lac de Grand-Lieu ; 1 : eau libre ; 2 : végétation palustre (macrophytes flottants); 3 : roselières boisées ; 4 : prairies humides et végétation riveraines. (d'après Boret et Reeber 2008, simplifié).

En termes d'écosystèmes, l'une des principales richesses patrimoniales du lac est constituée par des ceintures de macrophytes flottants très originales (GADECEAU, 1909 ; MARION, 1999 ; DUPONT, 2003) qui occupaient au milieu de $X^{\text {ème }}$ siècle une partie significative de la surface lacustre (figure 2). Or la densité et la surface de ces ceintures ont diminué de façon drastique pendant la seconde moitié du $\mathrm{XX}^{\text {ème }}$ siècle 
(perte de surface de près de 30\% sur la période 1975-1995). Cette diminution a affecté essentiellement les associations végétales les plus remarquables; ainsi à titre d'exemple, entre 1982 et 1996, on note la perte de 60\% de la surface des châtaignes d'eau Trapa natans, 70\% des limnanthèmes jaunes Nymphoides peltata (MARION, 1999 ; SNPN, 2009). Parallèlement, on a assisté à la disparition d'herbiers aquatiques remarquables (characées), à une plus grande fréquence des blooms algaires (microphytes) et à une augmentation corrélative de la turbidité des eaux. Le gestionnaire de l'époque a proposé et a mis en œuvre entre 1995 et 2000, des interventions lourdes financées par le programme européen "Life". Ce "plan de sauvetage du lac" (MARION, 1999) était explicitement basé sur des hypothèses qui reposent sur les modèles classiques d'évolution trophique des zones lacustres, à savoir : l'eutrophisation du milieu et le comblement du lac (la vitesse d'envasement admise à cette époque se situait entre 1 et 3 cm/an ; MARION \& MARION, 1976 ; JIGOREL, 1992 ; MARION, 1992 ; COMMISSARIAT AU PLAN, 1994, p. 298).
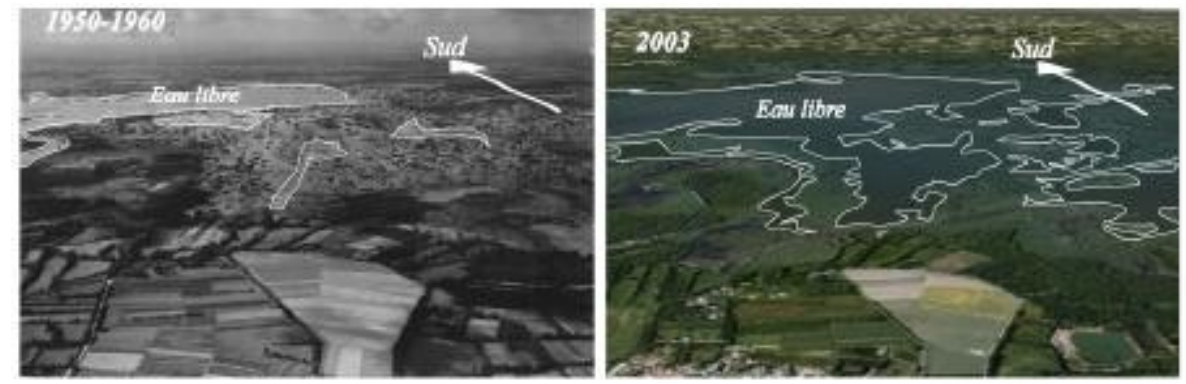

Figure 2. Comparaison d'une photographie aérienne oblique des années 1950 et d'une scène GoogleEarth qui montre l'augmentation de l'eau libre et le remplacement des herbiers à Scirpes lacustres (Scirpus lacustris), Limnanthèmes jaunes (Nymphoides peltata), et châtaignes d'eau (Trapa natans) par des herbiers à nénuphars blancs

(Nymphaea candida) et nénuphars jaunes (Nuphar luteum).

La mise en œuvre de ce plan n’a pas apporté les résultats escomptés et, entre 2000 et 2007, on a déploré une nouvelle régression la surface des ceintures de macrophytes flottants de plus de 10\%, mais surtout une accélération de la disparition des associations végétales les plus remarquables avec, par rapport à la situation de 1996, une nouvelle baisse de $85 \%$ des châtaignes d'eau Trapa natans, 79\% des limnanthèmes jaunes Nymphoides peltata (BORET \& REEBER, 2008). Par ailleurs, aucune évolution positive de la turbidité des eaux n’a pu être mise en évidence et de nouveaux problèmes préoccupants sont apparus, comme la régression des roselières (SNPN, 2009).

Face à cette situation, et à partir de 2005, la SNPN a réalisé ou soutenu des études visant à mieux comprendre la dynamique du milieu et notamment des facteurs abiotiques. C'est la synthèse de ces travaux que nous nous proposons d'exposer ici. Dans ce qui va suivre, nous voudrions montrer que la dynamique du milieu est 
Trois siècles d'histoire hydro-sédimentaire et écologique du lac de Grand-Lieu, conséquences pour la gestion hydraulique : 6.5

beaucoup plus complexe que celle qui fut postulée lors de l'élaboration du "plan de sauvetage", notamment du fait d'une anthropisation ancienne. Après une présentation du contexte hydraulique, nous discuterons en particulier de :

(i) l'évolution séculaire de l'épaisseur de la tranche d'eau, notamment au printemps, cette caractéristique étant un paramètre majeur de contrôle des écosystèmes de lacs peu profonds (SCHEFFER, 1998)

(ii) de la qualité chimique et physique (turbidité) des eaux, autre paramètre fondamental. (SCHEFFER, 1998)

En conclusion, nous montrerons que la prise en compte de ces données conduit à remettre fortement en question la gestion actuelle et à proposer des pistes pour l'élaboration d'une nouvelle gestion.

\subsection{Contexte hydraulique actuel}

Sur le plan morphologique, la cuvette de Grand-lieu correspond à une zone de convergence de trois rivières (Tenu, Boulogne et Ognon) juste en amont d'un rétrécissement de la vallée où s'enfile l'exécutoire du lac : l'Acheneau. Ce dernier se jette dans l'estuaire de la Loire (figure 3). L'altitude et la pente de la vallée de l'Acheneau sont extrêmement faibles, si bien qu'avant les travaux hydrauliques réalisés au XIX ${ }^{\text {ème }}$ siècle, les marées se faisaient sentir au niveau même du lac. Par ailleurs, à la même époque, des travaux ont permis la connexion directe du Tenu avec l'Acheneau si bien qu'actuellement seuls les bassins versants de la Boulogne et de l'Ognon (soit $840 \mathrm{~km}^{2}$ ) alimentent le lac.

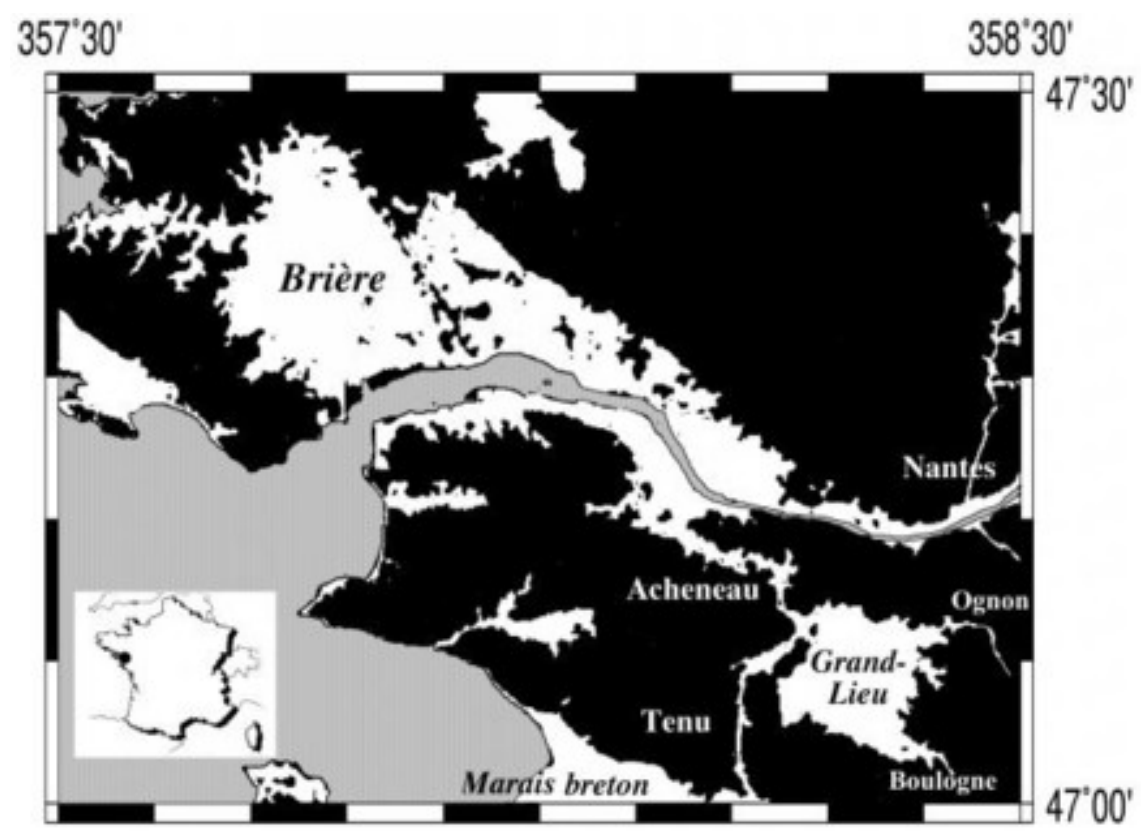

Figure 3. La zone humide de Grand-Lieu dans son contexte morphologique (en blanc : altitude inférieure à 2,5 m NGF). 
Le régime de ces rivières est très irrégulier (figure 4) et caractérisé par des débits quasiment nuls de juin à septembre alors qu'ils peuvent dépasser $90 \mathrm{~m}^{3} / \mathrm{s}$ lors des crues hivernales. Le niveau du lac et sa surface sont, de ce fait, eux-mêmes très variables dans l'année. En été sa surface lacustre est d'environ 1800 h et la profondeur moyenne est maintenue à 0,70 m environ grâce à l'écluse de Bouaye (figure 5). En hiver, et en dépit de l'ouverture de l'écluse, le niveau monte en moyenne d'un mètre et le lac déborde sur les roselières et les prairies humides riveraines et constitue un plan d'eau temporaire de plus de 6000 hectares (figure 1).

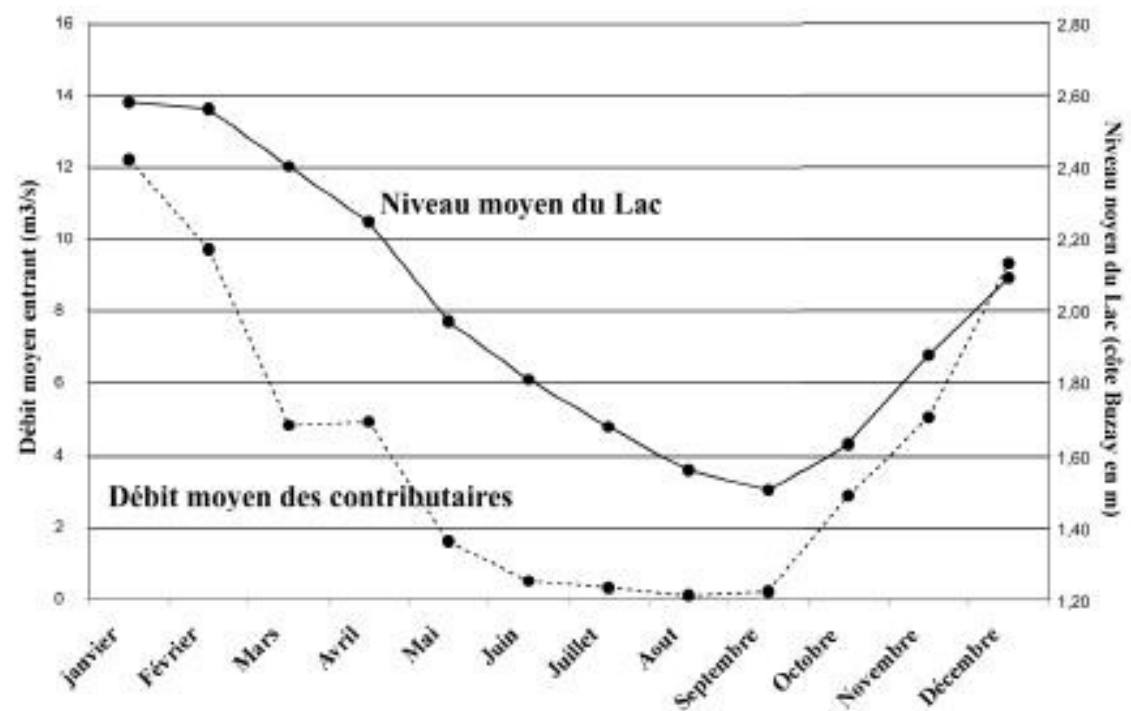

Figure 4. Moyenne inter-annuelle (1981-1994) du débit liquide de surface alimentant le lac et niveau moyen du lac (1981-1994). Ce niveau est donné, comme toutes les cotes citées dans ce travail, en cote "Buzay" (= NGF(69) - 0,45 m) qui est utilisée systématiquement comme référence hydraulique dans la région; Peltier, 2004). Cette cote correspond à l'ancien radier de l'écluse de Buzay.

\section{Histoire de la tranche d'eau}

Dans les lacs peu profonds, l'épaisseur de la tranche d'eau, notamment au printemps, est un paramètre fondamental de l'écosystème aquatique (SCHEFFER, 1998). Cette épaisseur correspond à la différence entre l'altitude du niveau d'eau, et l'altitude de l'interface eau/sédiments dont la dynamique propre résulte du bilan érosion/sédimentation. Nous envisagerons successivement ces deux points dans leur dimension historique depuis le début du XVIII ${ }^{\mathrm{ème}}$ siècle.

\subsection{Evolution historique des niveaux d'eau}

Avant le milieu du $\mathrm{XX}^{\text {ème }}$ siècle, l'exploitation agricole des marais au printemps (par fauchage et pâturage) était très importante pour les riverains des zones humides. Cette 
Trois siècles d'histoire hydro-sédimentaire et écologique du lac de Grand-Lieu, conséquences pour la gestion hydraulique : 6.7

exploitation n'était possible que si l'évacuation de la crue hivernale intervenait tôt en saison. Il apparaît que cela n’a pas été toujours le cas dans la région de Grand-Lieu. Ainsi, le Sieur Boussineau, qui a visité le lac en 1712, dans le cadre d'une mission officielle, signale que le lac était encore en crue en juin et que cette crue durait, sans discontinuité, depuis sept ans (BOURRIGAUD, 2007). Ce genre d'inondation transannuelles semble avoir été très fréquent dans le passé ; les riverains et les autorités ont donc essayé de tout temps de favoriser les écoulements à l'aval du lac. Une délibération du Conseil ducal de 1460 constitue la référence historique la plus ancienne (LESCADIEU \& LAURANT, 1836). Cette volonté s'est heurtée aux conditions naturelles à l'aval du lac qui sont très défavorables: l'Acheneau est une rivière à la pente quasiment nulle, dont le flux s'inversait deux fois par jour à cause de la marée. Par ailleurs son cours était encombré par un colmatage naturel et de nombreux barrages artificiels (les pêcheries et autres moulins du Pilon sont, à ce titre, rentrés dans l'histoire ; PIERRELEE \& LAMPRIER, 2004).
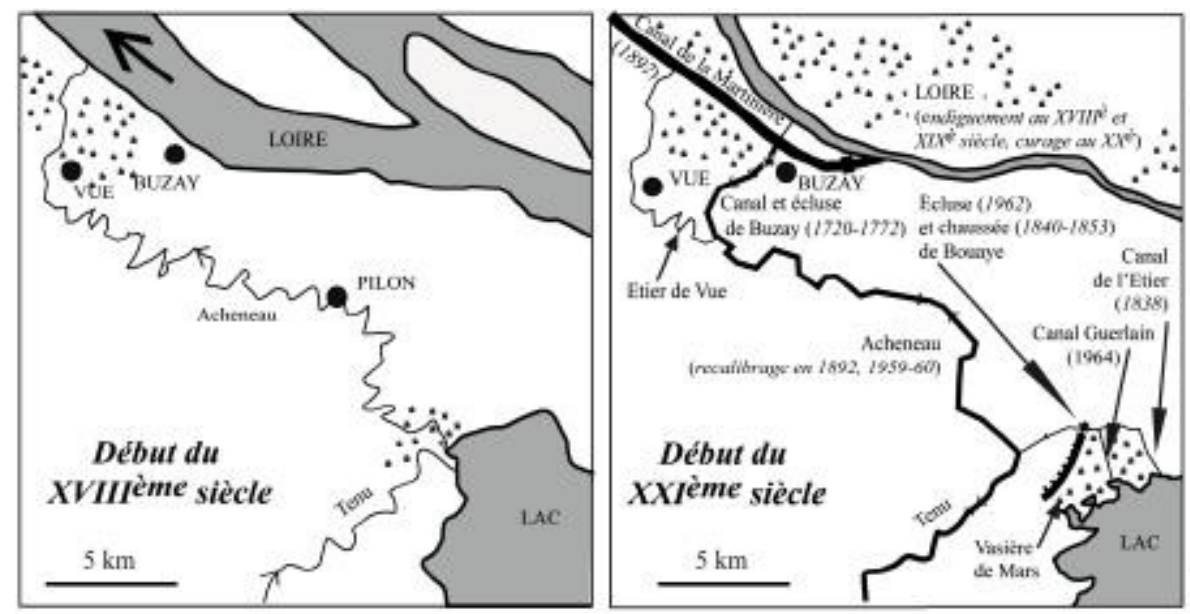

Figure 5. Schéma des aménagements hydrauliques à l'aval du lac de Grand-Lieu depuis le début du XVIII ${ }^{\text {ème }}$ siècle.

Ce n'est qu'au début du XVIII ${ }^{\text {ìme }}$ siècle que la technique, le contexte économique et les circonstances politiques, permettront la mise en place d'un début de solution avec la constitution, vers 1720, de la "Société des intéressés au dessèchement". Celle-ci se donne pour but la réalisation du canal de Buzay (figure 5) qui constitue un raccourci hydraulique entre le lac et la Loire. Les travaux dureront longtemps (jusqu'en 1772 au moins) mais l'efficacité hydraulique semble avoir été atteinte en 1769. On trouve en effet dans les archives départementales de la Loire Atlantique un mémoire daté de 1782 (C123-P111) qui signale que "depuis 1769, les marais sont fauchés tous les ans; une ou deux fois, ce qui n'était pas le cas auparavant, sauf années extraordinairement sèches." ; un document des archives paroissiales de Sainte Lumine de Coutais et rédigé par le recteur de cette paroisse, confirme à la fois le phénomène et la date : "Cette année 
1769... nos marais se sont trouvés à sec à la saint Jean, malgré l'inondation extraordinaire qui a régné tout l'hiver...(le canal) de Buzay se met aussi à la perfection ce qui nous fait espérer que désormais nous jouirons de nos marais tous les ans" (Freor 1979, p 38). Selon MILLE (1859) ce sont 3000 hectares de prairies qui sont créés aux dépens des marais à cette époque, en amont du canal de Buzay. La carte de Cassini, livrée entre 1783 et 1786, qui est la première à figurer un réseau de drainage dans les "marais" de Sainte Lumine, témoigne de cette évolution de l’hydraulique et du paysage. Par la suite, des crues hivernales importantes vont encore avoir lieu (4,50 m en cote Buzay (voir légende de la figure 4 pour des explications sur cette cote), en Janvier 1853, 4,55 m en 1872 soit plus de $2 \mathrm{~m}$ au-dessus des cotes hivernales moyennes actuelles), mais, à chaque fois, la situation semble être redevenue normale au cours du printemps. Nous n'avons pas de témoignage direct de la cote estivale du lac à cette époque. Néanmoins, une cote officielle de navigation a été fixée pour le lac en 1830, il s’agit de 2,00 m en cote Buzay. On peut raisonnablement penser qu'elle est proche de la cote moyenne estivale, en effet, VALLES (1848) précise que l'ensemble Acheneau/lac était réalimenté par la Loire en marée montante quand le niveau descendait sous le seuil de 2 m Buzay.

Entre 1881 et 1892, de nouveaux travaux hydrauliques importants ont lieu (figure 5). Il s'agit du creusement du canal de la Martinière (VEZIER-VAUTHIER, 1992) ainsi que des travaux d'accompagnement sur l'Acheneau (MAITRE, 1912 ; 1912-1915). Bien qu'indépendants de tout projet hydraulique concernant le lac de Grand-Lieu, ces travaux ont un impact sur lui : c'est à cette époque que les marées cessent de se faire sentir dans le lac; mais surtout, on assiste à une nouvelle amélioration des conditions d'évacuation des crues hivernales. Par voie de conséquence, le niveau d'étiage baisse (GUILLOU et al., 2008); situation qu'entérine l'administration en diminuant le niveau de navigation (d'abord 1,75 m cote Buzay ; GUICHARD, 1887), puis 1,60 m : étiage dit de tolérance : GADECEAU, 1909). C’est aussi à cette époque que le Bonhomme, qui était antérieurement une île située au sud-est du lac (MANET, 1834) se raccorde à la côte (GADECEAU, 1909) (figure 1).

Logiquement la période qui suit (entre 1892 et 1958) est caractérisée par des niveaux estivaux bas. On note, à cette époque, une grande variabilité interannuelle qui reflète sans doute l'ampleur des crues hivernales. Une rupture semble intervenir à la fin des années 30 avec des niveaux encore plus bas et des fluctuations interannuelles limitées (figure 6). Les causes de cette rupture ne sont pas précisément connues ; elles peuvent correspondre à une évolution climatique (la période 1940-1960 est une période de relative sécheresse) ; et/ou à une modification de la gestion du canal de la Martinière rendue possible par une baisse du niveau des marées basses en Loire à partir de 1938 (figure 7). Toujours est-il que la période correspond aux niveaux printaniers les plus bas enregistrés. 
Trois siècles d'histoire hydro-sédimentaire et écologique du lac de Grand-Lieu, conséquences pour la gestion hydraulique : 6.9

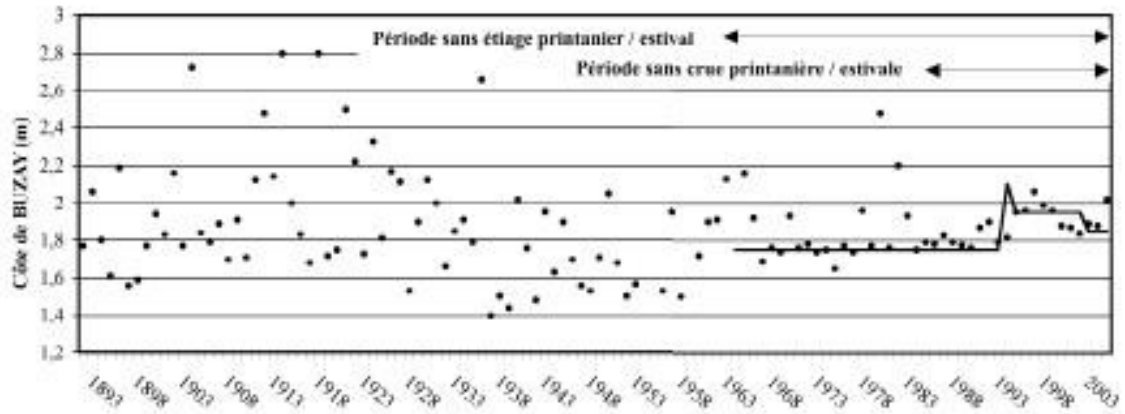

Figure 6. Cote du lac au 1er juin depuis 1893. Le trait gras continu indique l'évolution du règlement d'eau appliqué. On remarque deux ruptures principales dans la gestion qui correspondent à la construction de l'écluse de Bouaye, au début des années 1960, puis à une plus grande rigueur dans le respect du règlement d'eau (1985).

Entre 1958-1964, un profond recalibrage de l'Acheneau est réalisé (figure 5). Il s'agissait d'utiliser, en période estivale, cette rivière et le Tenu pour transférer, à contre pente naturelle, les eaux de la Loire vers le Marais Breton situé plus au sud (TALUREAU, 1965). Bien que ce ne soit pas le but recherché, ces travaux facilitent grandement l'évacuation des débits à la fin de l'hiver et au printemps. Par voie de conséquence les cotes du lac se sont écroulées spectaculairement, particulièrement à la fin de l'été. Le propriétaire du lac a alors obtenu la construction de l'écluse de Bouaye qui commandera le niveau du lac à partir de 1962.

La création de cette écluse répondait à la nécessité de garantir un niveau minimum dans le lac en été. Mais dès les premières années, elle fut utilisée pour remonter ces niveaux de façon à faciliter l'installation des canards dont la chasse constituait alors la principale fonction du lac. En pratique, le niveau moyen estival a été augmenté de presque $20 \mathrm{~cm}$ (GUILLOU et al., 2008) et les étiages estivaux supprimés (figure 6). Cette politique a été entérinée par l'administration (arrêté de 1965, PIERRELEE \& LAMPRIER, 2004). En pratique des cotes étaient fixées pour chaque début de mois de mars à septembre. En 1985, un opérateur spécialisé a pris en charge la gestion de l'écluse et cela s'est traduit par un respect beaucoup plus rigoureux de ces cotes (figure 6). Au total, (i) l'augmentation de la capacité structurelle à évacuer l'eau hivernale, (ii) le mode de gestion de l'écluse et (iii) des aménagements secondaires (creusement, en 1964, du canal Guerlain qui constitue un raccourci hydraulique entre le lac et l'écluse ; figure 5), concourent à augmenter de façon importante les pointes de courants de vidange dans le lac (figure 8). 


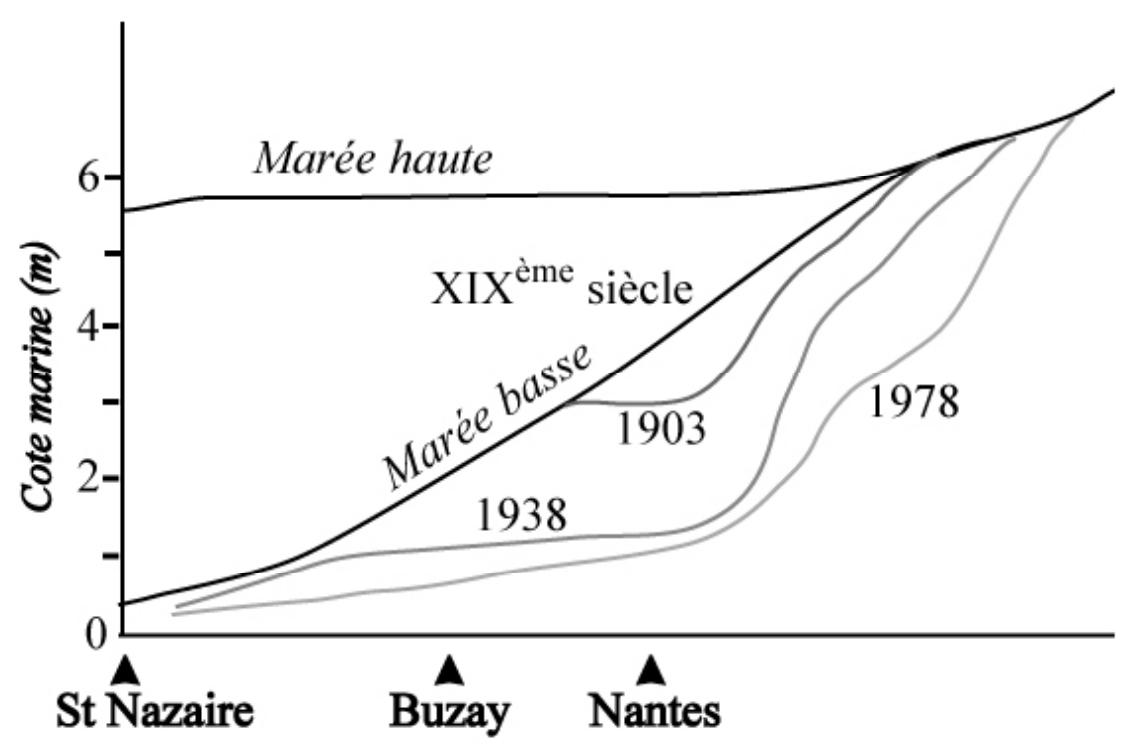

Figure 7. Evolution des lieux géographiques des marées hautes et basses dans l'estuaire de la Loire (conditions de vives-eaux et étiage) ; d'après Migniot et Le Hir, 1994, simplifié.

Depuis 1995 et suite à l'élaboration d'une doctrine de gestion directement issue du "plan de sauvetage" évoqué plus haut, un nouvel arrêté a remonté les cotes (+0,50 m au 1er avril par exemple). Des conflits importants avec les riverains étant apparus, de nouvelles dispositions modérant la hausse de niveau sont prises successivement en 1996 et 2004 (PIERRELEE \& LAMPRIER, 2004).

\subsection{Evolution historique de la dynamique érosion/sédimentation}

En constituant des plans d'eau horizontaux, les lacs forment des anomalies par rapport aux profils d'équilibre des rivières, ils sont donc très naturellement condamnés à se combler. Dans les lacs peu profonds, ce comblement se traduit à la fois par un rehaussement des fonds et par un envasement des rives. En l'absence de campagnes anciennes de bathymétrie, la comparaison des cartes anciennes est un moyen privilégié pour juger de la dynamique sédimentaire globale.

Les cartes antérieures au XVIII ${ }^{\text {ème }}$ siècle apparaissent trop imprécises, voire manifestement trop éloignées de la réalité, pour être utilisées dans notre analyse. 


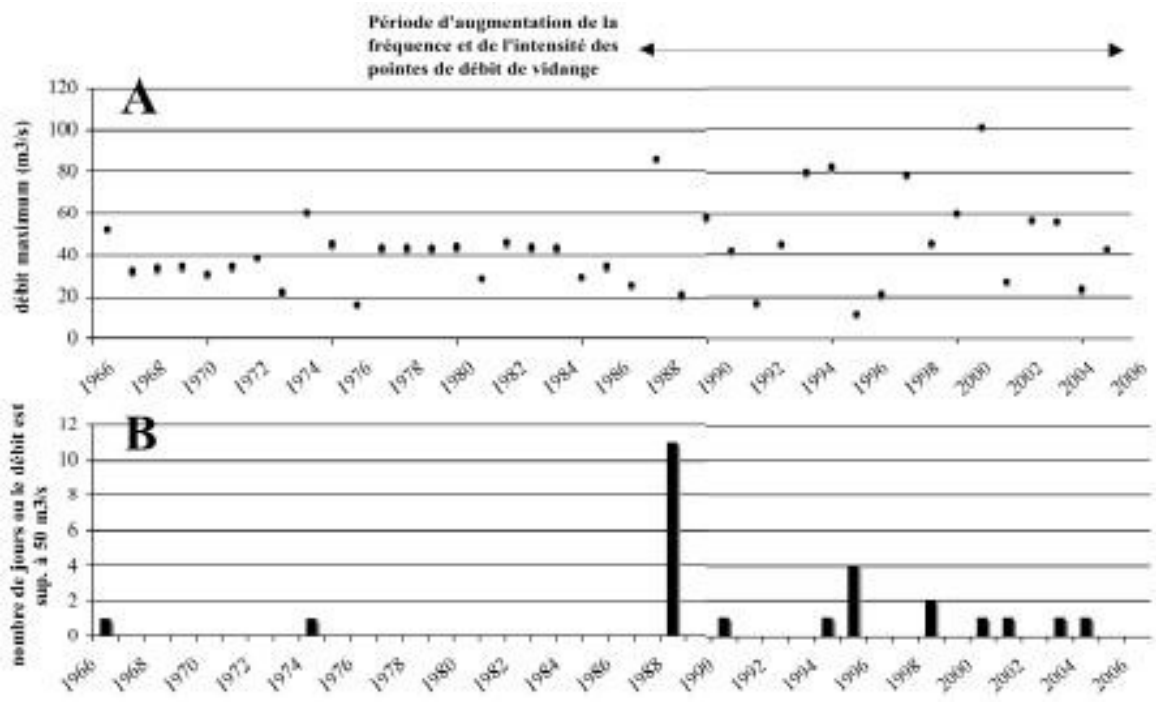

Figure 8. A : Maxima annuels des débits journaliers en sortie de lac, et B : Nombre de jours par an où le débit journalier est supérieur à $50 \mathrm{~m}^{3} / \mathrm{s}$.

Les premières cartes crédibles sont celles de: De Lambilly datant de la dernière décennie du XVII ${ }^{\text {ème }}$ siècle ; et celle de 1713 (figure 9). Elles nous présentent un lac bordé sur sa façade ouest et sud-ouest par une zone de marécage. Le sieur Boussineau, déjà mentionné, qui a visité le lac à cette époque donne suffisamment d'éléments de bathymétrie pour calculer, qu'en dehors de la crue, la profondeur était de l'ordre de 0,3 à 1,3 m dans les chenaux de navigation (BOURRIGAUD, 2007 ; GUILLOU et al., 2008). Le lac de l'époque était donc, sur le plan de sa surface comme de sa profondeur, très comparable à l'actuel. Par ailleurs, les processus d'envasement étaient suffisamment évidents pour que, lorsque Boussineau a demandé aux propriétaires des marais la communication des surfaces de leurs biens; il lui a été répondu que les chiffres qui apparaissent dans les actes officiels étaient inexacts du fait des "acquêts", c’est-àdire de l'extension des surfaces liées à l'envasement (BOURRIGAUD, 2007).

Les cartes de 1750 (reproduite in PIERRELEE \& LAMPRIER, 2004, p. 144) et 1754 (reproduite in PIERRELEE \& LAMPRIER, 2004, p. 137) semblent indiquer que la surface des marais continue à augmenter bien que la précision de ces documents ne permette pas d'être absolument affirmatif. 


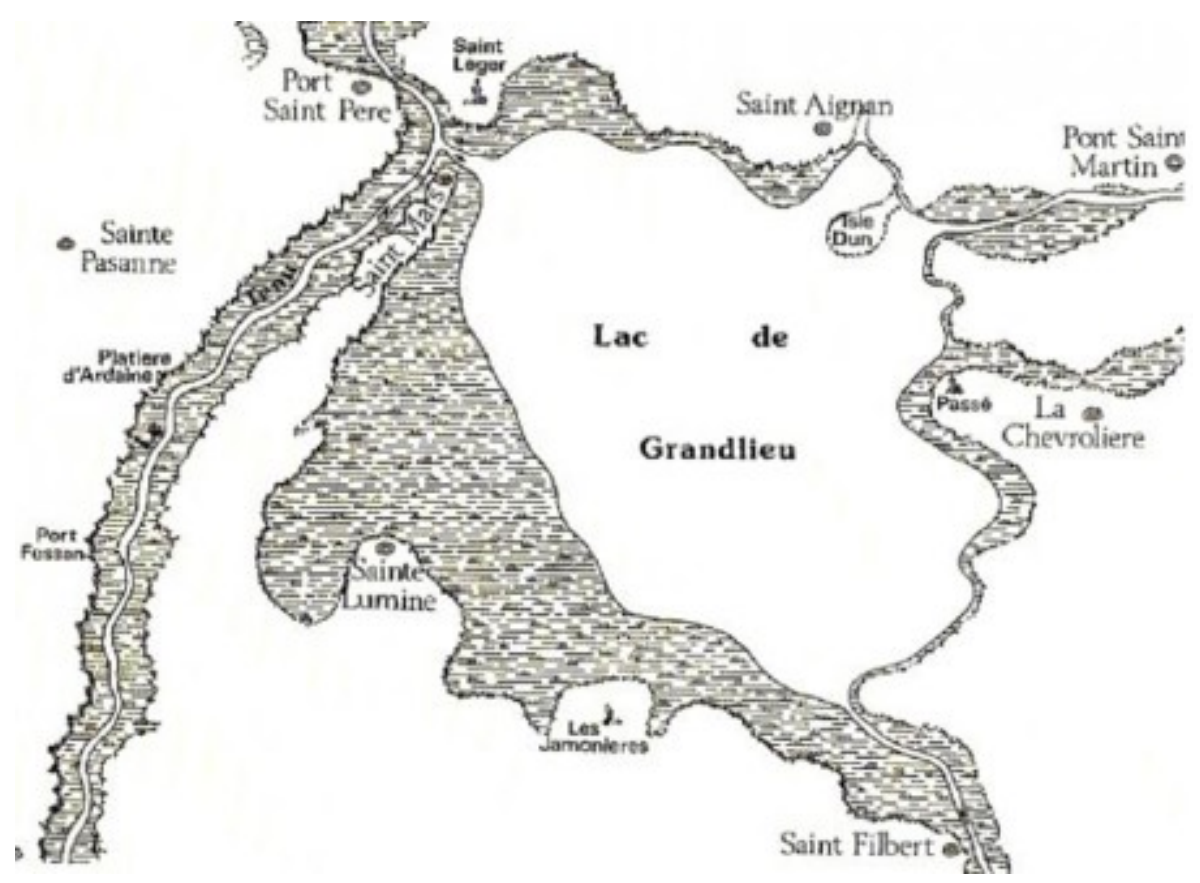

Figure 9. Carte du lac de Grand-Lieu en 1713 (extrait), on remarque que l'importance de la zone lacustre est très proche de l'actuelle. Par ailleurs, les futures prairies de St

Lumine, au sud-ouest du lac (voir figure 1), sont encore indiquées en marécages, la transformation en prairies ne sera effective qu'après l'ouverture du canal de Buzay et la baisse de niveau corrélative.

Après la réalisation du canal de Buzay, la carte de Cassini, établie entre 1783 et 1786, celle de Ballays (1786, reproduite in PIERRELEE \& LAMPRIER, 2004, p.139) et celle de Juigné (1812, reproduite in PIERRELEE \& LAMPRIER, 2004, p.168) nous montrent un lac totalement dépourvu de marais en rive. En marge de la seconde carte, il est indiqué qu'au mois de Juillet 1786 (c'est à dire en étiage) la surface du lac était de : "7779 journaux, 62 cordes et 13/16 de cordes", mesures de Bretagne, soit 3763 hectares (pour l'équivalence des unités, voir PELIEU, 1852) ce qui est le double de la surface actuelle en période comparable. Entre 1754 et 1786, c'est-à-dire lors de la mise en eau du canal de Buzay, un processus d'érosion des rives marécageuses du lac a manifestement eu lieu.

Un demi-siècle après, vers 1838, l'envasement semble avoir repris, le lac fait alors 3600 hectares en été (VALLES, 1848). En 1859, la première campagne bathymétrique complète du Lac, par ailleurs calée sur la cote Buzay, est réalisée (MILLE, 1859). Nous disposons du graphique hypsométrique correspondant (figure 10). En accord avec cette figure, l'auteur précise sans ambiguïté que "en étiage, quand l'eau est tenue à 1,70m, il y a une plaine liquide de 3400 hectares..."). Ce dernier signale par ailleurs que "l'envasement progressif de la rive gauche, où le colmatage se fait à l'abri du vent de S.O., et où la plaine de roseaux gagne parfois 100 m en une année...". 
Trois siècles d'histoire hydro-sédimentaire et écologique du lac de Grand-Lieu, conséquences pour la gestion hydraulique : 6.13

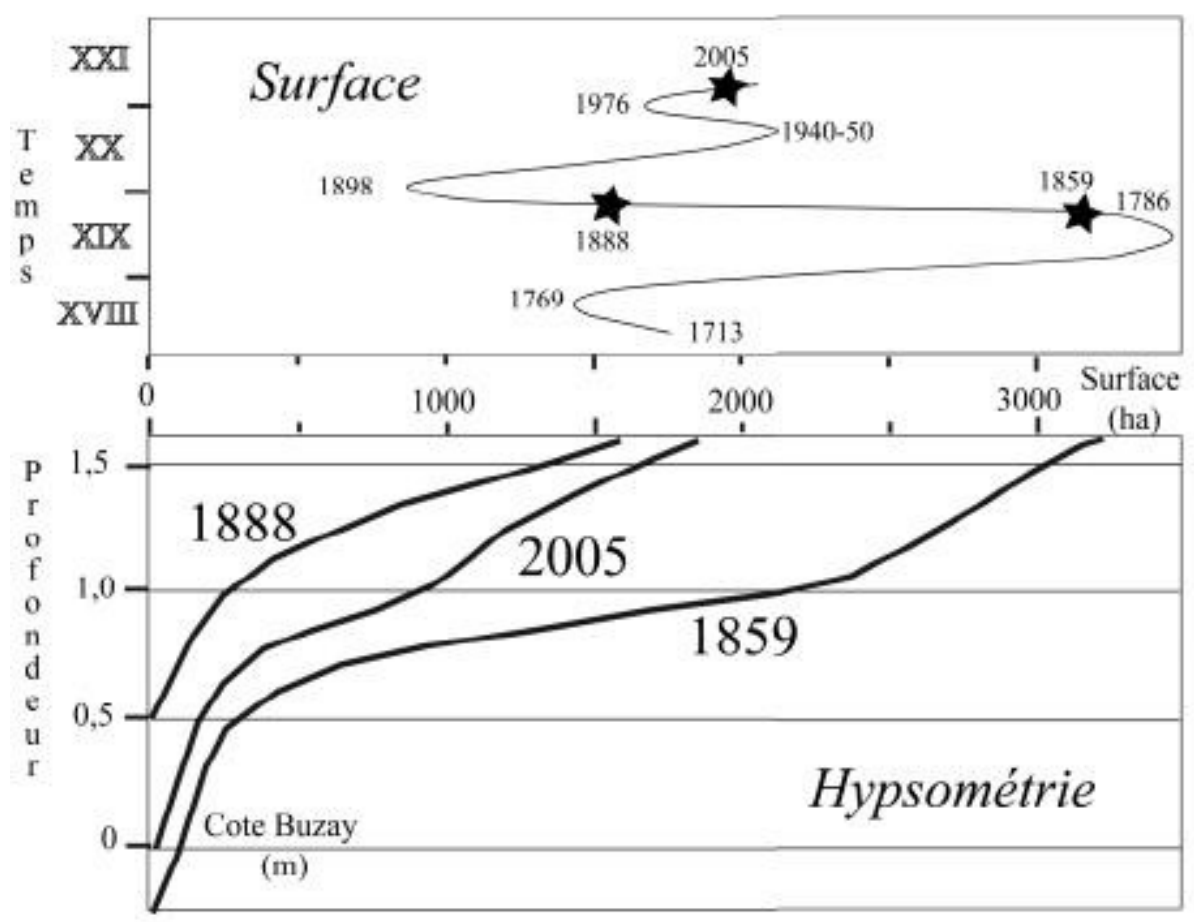

Figure 10. Courbes hypsométriques du lac en 1859 (MILLE, 1859) ; 1888 (PINSON, 1888) ; et 2005 (BORET, 2005). Ces courbes sont localisées par des étoiles dans la représentation des alternances augmentation / diminution de la surface lacustre documentées depuis le début du XVIII ${ }^{\text {ème }}$ siècle.

Il est probable que la percée du canal de l’Etier (1838) et la réalisation de la chaussée de Bouaye (à partir de 1840), qui imposent un allongement du chemin hydraulique entre le lac et la Loire, sont des facteurs favorables au développement de la vasière de Mars et, plus généralement, à l'envasement du lac. En 1888, une nouvelle campagne bathymétrique calée sur la cote Buzay est réalisée (PINSON, 1888, figure 10). On constate que la surface du lac a fortement diminué (1700 hectares) et que les sédiments se sont accumulés, depuis 1859, avec un taux de sédimentation proche de $2 \mathrm{~cm} / \mathrm{an}$. C'est vers cette époque que les études scientifiques sur le milieu naturel commencent à se généraliser, les auteurs de cette époque (LLOYD, 1876; GUICHARD, 1887 ; DELEBECQUE, 1898) s'accordent à souligner l'envasement très rapide qui affecte le lac de Grand-Lieu.

Dans les années qui suivent immédiatement l'ouverture du Canal de la Martinière (1892), l'accumulation des vases, qui s'est probablement poursuivie, et la baisse du niveau d'étiage concordent à réduire fortement la surface du lac. Ainsi, A. Lavéran, futur Prix Nobel pour ses travaux sur le paludisme, qui a travaillé à Grand-Lieu à cette époque, indique "j'ai constaté qu'il s'était transformé en marais sur la plus grande partie de son étendue." (LAVERAN, 1898). Par ailleurs une carte établie entre 1894 et 
1899 (ANONYME 1894 à 1898, reproduite in PIERRELEE \& LAMPRIER, 2004, p.179) représente une zone lacustre ne dépassant pas 1000 hectares.

Dans les premières années du XX ${ }^{\text {ème }}$ siècle GADECEAU (1909) a étudié la végétation de la zone humide de Grand-Lieu. Ayant, à cette occasion, réalisé quelques mesures de profondeur, il souligne que les données antérieures dont il dispose (bathymétrie partielle de GUICHARD, 1887) sont sous-évaluées, il va même jusqu’à rajouter sur la carte de cet auteur, qu'il re-publie, des profondeurs supplémentaires, toutes largement supérieures à celles indiquées sur la carte originale. Alors que le niveau d'eau est plus bas, cette augmentation de la profondeur des fonds témoigne de la reprise de l'érosion. Cette phase érosive suit donc, mais avec quelques années de retard, la baisse de niveau consécutive aux travaux hydrauliques réalisés en aval.

La comparaison des cartes de l'époque avec la campagne de photographies aériennes de 1945 montre que cette phase d'érosion est marquée par le démantèlement de l’immense roselière qui s'était constituée à l'ouest du lac à la fin du XIX ${ }^{\text {ème }}$ siècle. Les grandes îles couvertes de roselières boisées visibles sur la figure 1 constituent des témoins de ce recul. Les campagnes de photographie suivantes révèlent que la période 1945/1950 marque la fin du cycle érosif et le début d'une nouvelle phase de diminution de la surface du Lac. C'est au cours de cette nouvelle période d'atterrissement qu'intervient la construction de l'écluse de Bouaye et la remontée des niveaux.

D’après le suivi des photos aériennes, la phase d'engraissement des marais s'est poursuivie jusqu'en 1976 (GUILLOU et al., 2008). À cette date, la tendance s'inverse avec d'abord un surcreusement spontané du canal Guerlain qui voit sa largeur passer de 6 à 25 m, puis par l'apparition de berges en régression au Nord du Lac, c'est-à-dire à proximité de ses exutoires. Depuis 1979, ce phénomène s’est généralisé. En 1997 et en 2005, la SNPN a réalisé deux campagnes de bathymétrie (plus de 2000 points mesurés deux fois à 8 ans d'intervalle, BORET, 2005). La courbe hypsométrique de la plus récente est indiquée sur le graphique de la figure 10. Ces campagnes ont permis de chiffrer le recul des roselières boisées en 8 ans (45 hectares) et l'approfondissement corrélatif des fonds (environ 0,5 cm). Les derniers relevés de surface suggèrent une accélération du phénomène avec des pertes annuelles de l'ordre de 15 à 20 hectares (MERCIER \& SANCHEZ, 2010a).

\section{Histoire de la qualité des eaux}

Avec l'épaisseur de la tranche d'eau, la qualité des eaux est le principal paramètre abiotique qui contrôle la qualité d'un milieu lacustre/palustre. Deux aspects semblent prépondérants : la teneur en nutriments (azote et phosphore, respectivement sous forme de nitrate et phosphate), et la turbidité des eaux qui reflète, dans les lacs de plaine, la quantité de phytoplancton. 
Trois siècles d'histoire hydro-sédimentaire et écologique du lac de Grand-Lieu, conséquences pour la gestion hydraulique : 6.15

\subsection{Aspect chimique}

Nous disposons de l'analyse d'une eau prélevée le 7 juillet 1851 (ANONYME, 1851 ; HERY-VIOLETTE \& ARCHAMBAULT, 1859), les nutriments ne sont pas mesurés mais la qualité globale ne devait déjà pas être considérée comme satisfaisante car CONSTANTIN \& RODET (1892) précisent: "ce ne sont donc point des eaux à recommander". MARION \& MARION (1976) font référence à deux analyses d'avril 1972 et indiquent que les nitrates sont à l'état de trace. Les analyses ponctuelles les plus récentes sont celles de l'Agence de Bassin dont un récapitulatif, en ce qui concerne les nutriments, est donné en figure 11. Ces chiffres indiquent une mauvaise qualité de l'eau, surtout en été. Signalons également l'installation récente d'espèces végétales tolérant la pollution de l'eau (DUPONT, 2003).

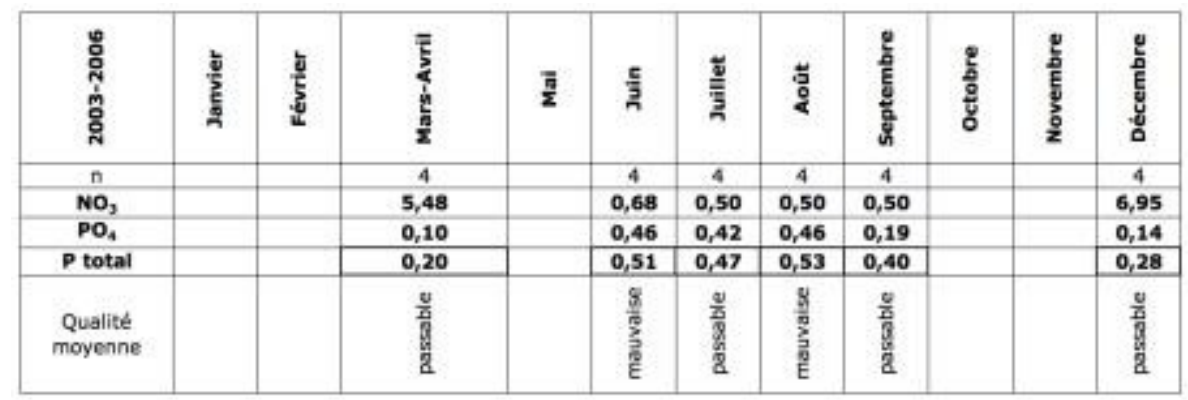

Figure 11. Moyennes interannuelles (2003-2006) des teneurs en nutriments du lac (mesures en mg/l de l'Agence de Bassin). La qualité moyenne est appréciée à partir des teneurs moyennes en nutriments et en retenant le paramètre le plus pénalisant au regard des seuils du système normalisé SEQ-eau (Anonyme, 1999). C'est le phosphore qui constitue le paramètre le plus pénalisant et, comme c'est souvent le cas, qui contrôle les processus d'eutrophisation.

\subsection{Histoire de la turbidité des eaux}

Le tableau de la figure 12 résume les observations des savants et des voyageurs qui ont décrit l'aspect des eaux du lac de Grand-Lieu aux XVIII et XIX ${ }^{\text {ème }}$ siècles. Le fait que certaines expressions soient redondantes suggère que certaines sources ne sont pas de première main... On remarque néanmoins une quasi-unanimité pour décrire des eaux turbides. La seule exception (GIRAULT DE SAINT-FARGEAU, 1829a ; 1829b) est à relativiser dans la mesure où le même auteur décrit des eaux troubles en avril 1826, et surtout que son affirmation est explicitement mise en doute par MANET (1834) quelques années après. On note par ailleurs qu'au moins trois de ces références concernent des observations effectuées durant le printemps ou l'été (GIRAULT DE SAINT-FARGEAU, 1829b ; VAYSSE DE VILLERS, 1831 ; ANONYME 1851 ). 


\begin{tabular}{|c|c|}
\hline "... le Lac de Grand-Lieu retient des eaux vaseuses..." & Barron (1899) \\
\hline "... le Lac de Grand-Lieu retient des eaux vaseuses..." & Baron (1888) \\
\hline "Grand-Lieu est un lac noir et bourbeux ..." & Dugast-Matifeux (1879) \\
\hline $\begin{array}{l}\text { "Ce réservoir... contient ...de l'eau trouble d'odeur } \\
\text { marécageuse..." }\end{array}$ & Lefort (1859) \\
\hline $\begin{array}{l}\text { ".... (le } 7 \text { juillet) ... eaux troubles, exhalant une légère odeur } \\
\text { de marécage et donnant un dépôt très sensible..." }\end{array}$ & $\begin{array}{l}\text { Anonyme (commission spéciale) } \\
\text { (1851) }\end{array}$ \\
\hline $\begin{array}{l}\text { "... le Lac de Grand-Lieu...ses eaux sont noiratres et } \\
\text { bourbeuses." }\end{array}$ & Déric (1847) \\
\hline $\begin{array}{l}\text { "M. Girault affirme que c'est une belle nappe d'eau limpide, } \\
\text { M. de Robien, au contraire et plusieurs autres, assurent que } \\
\text { ses eaux sont presque toujours troubles." }\end{array}$ & Manet (1834) \\
\hline $\begin{array}{l}\text {... Acheneau ... n'a pas de cours...(son eau) dormante et } \\
\text { bourbeuse, comme celle du Lac même" "... defaut de } \\
\text { limpidité des ... eaux (du Lac) .... }\end{array}$ & Vaysse de Villers (1831) \\
\hline $\begin{array}{l}\text { "... en avril } 1826 \text {...les eaux de lac, très agitées, nous } \\
\text { paraissent troubles.." }\end{array}$ & Girault de Saint-Fargeau (1829b) \\
\hline "...Lac de Grand-Lieu, belle nappe d'eau limpide.." & Girault de Saint-Fargeau (1829a) \\
\hline "....un grand lac noir et bourbeux..." & de Voyer Argenson (1783) \\
\hline "Grand-Lieu est un lac noir et bourbeux..." & Pignaniol de la Force (1754) \\
\hline
\end{tabular}

Figure 12. Témoignages datant des XVIII et XIX $X^{\text {ème }}$ siècles concernant l'aspect des eauX du lac de Grand-Lieu.

Lloyd, le célèbre botaniste, a étudié la végétation du lac et a donné un compte-rendu de ses observations tout d'abord dans l'ouvrage intitulé "une flore de la Loire inférieure" (LLOYD, 1844) qui a été réédité ensuite, avec de nombreux ajouts et corrections et une expansion de l'aire d'étude, en 1854, 1869, 1876, 1886 et 1897. Au total ces ouvrages constituent un suivi de l'évolution du lac de Grand-Lieu pendant toute la seconde moitié du XIX ${ }^{\text {ème }}$ siècle. En 1844, Lloyd, indique que "le lac de Grand-Lieu ... n'offre pas au botaniste tout l'intérêt qu'il désire rencontrer...", "..il y croît... les mêmes plantes que dans de nombreux marais de ce département" ; avec le vocabulaire actuel, on dirait que la biodiversité y est faible et ne présente pas d'intérêt particulier !. La description est la même en 1854, et en 1876 il signale, de plus, un envasement rapide et il pronostique la disparition prochaine du lac (voir § précédent). Ce n’est que dans l'édition de 1897 (p. LXX) qu'il manifeste un tout autre intérêt pour l'écosystème du lac "... la localité (le lac) mérite d'être détaillée. J'ai exploré le lac dans tous les sens et suivi tous ses bords..." ; dans les paragraphes qui suivent, il décrit longuement les différents milieux qu'il a observés et à cette occasion, il insiste sur l'existence de vastes et denses herbiers de characées. Or, on considère ces characées comme de bons bio-indicateurs de la qualité de l'eau du point de vue de l'absence de turbidité et de l'oligotrophie. Le milieu semble donc avoir radicalement changé en ces dernières années du XIX ${ }^{\text {ème }}$ siècle ; notamment en ce qui concerne la turbidité des eaux.

Cette absence de turbidité est confirmée par GADECEAU (1909), autre botaniste célèbre, qui a réalisé des tests au disque de Secchi (15 juillet $1906: 1,45 \mathrm{~m}, 30$ Septembre 1906: 1,50 m). Cet auteur réalise par ailleurs, à partir de 1898, de nombreuses découvertes botaniques (DUPONT, 2003) ce qui confirme la récente modification des conditions du milieu. Dans les années suivantes, et au moins jusqu'à la fin des années 1950, la transparence de l'eau, notamment en été et au printemps, est la 
règle. On dispose, à ce sujet, de nombreux témoignages de riverains grâce au travail à caractère ethnographique d'ANDRE et al. (2000). Selon DUPONT (2003) la période 1958-1966 correspond à la période de disparition des espèces végétales oligotrophes emblématiques du lac. MARION \& MARION (1976) mentionnent 6 tests au disque de Secchi, essentiellement au mois d'août, qui donnent des profondeurs de 0,20 à 1,20 m (moyenne $(0,55 \mathrm{~m})$. Par ailleurs sur les 6 espèces de charas signalées dans le lac par les auteurs précédents, 4 sont considérées comme "vraisemblablement disparues", ou "à rechercher". Actuellement les characées ont totalement disparu, et les eaux sont troubles en permanence comme en témoignent les 77 mesures de BRUNAUD (2007) au printemps 2006 qui ne dépassent nulle part 0,56 m avec une moyenne de 0,32 m.

\section{Discussions}

Il ressort de ce qui précède que l'évolution des paramètres abiotiques du milieu, notamment l'épaisseur de la tranche d'eau, et la qualité des eaux, est extrêmement complexe et a été fortement influencée par une anthropisation précoce du milieu. Il était classiquement postulé (MARION, 1999) : (i) que l'envasement continu est la règle depuis le XVIII ${ }^{\text {ème }}$ siècle, (ii) que le colmatage de la zone lacustre est imminent, (iii) que l'épaisseur minimum de la tranche d'eau a été atteinte dans les années 1990, et (iv) que la turbidité élevée des eaux est la conséquence unique des apports polluants "modernes" du bassin versant ; les résultats présentés précédemment ne vont pas dans ce sens.

\subsection{La question de l'envasement et des crises érosives}

Il apparaît que le lac est soumis à des cycles d'envasement/érosion qui sont contrôlés par les aménagements hydrauliques. En dehors de tout phénomène d'érosion des sédiments lacustres, MERCIER \& SANCHEZ (2010a) ont montré que les apports allochtones (matières en suspension -MES- venant du bassin versant) et autochtones (sédimentation organique lacustre) étaient actuellement très faibles (équivalents à une couche de vase de 0,6 à 2,1 mm d'épaisseur par an). Or la deuxième moitié du XIX ème siècle correspond à une intense période d'envasement du lac de Grand-lieu que l'on peut chiffrer à environ $2 \mathrm{~cm} / \mathrm{an}$. A cette époque, un facteur, inactif actuellement, est intervenu pour doper l'accumulation de vase. Nous pensons qu'il peut s'agir de l'érosion des terres agricoles. Le $\mathrm{XIX}^{\mathrm{ème}}$ siècle est en effet une période d'intense conquête de nouveaux espaces agricoles dans la région (RIEFFEL, 1834); ainsi en1855, il reste encore 100000 hectares de landes dans le département (PITRE AUBINAIS, 1855), mais en 1862, le commisse de Saint Philibert constate "que, dans tout le canton, il n’y a plus ni lande ni bruyère" (cité par NOTHOMMES 2005). Une preuve directe de cet alluvionnement accru peut être trouvée dans la carte bathymétrique de 1888 et dans 
la carte de 1898 qui montre la présence, à cette époque d’une barre de vases décantées perpendiculaire au courant, au débouché de l'estuaire de l'Ognon.

La création des canaux de Buzay et de la Martinière, ainsi que les travaux de recalibrage massif de l'Acheneau de la fin des années 1950, sont des éléments déclencheurs de crises érosives. L'analyse de la crise érosive actuelle montre que ce n'est pas simplement la baisse du niveau de base qui provoque, comme cela est classique, une érosion régressive. En effet, cette crise débute après la création de l'écluse de Bouaye, alors que le niveau d'eau était plus haut que pendant la période d'alluvionnement antérieure. MERCIER \& SANCHEZ (2010a) ont proposé, à partir d'arguments hydrauliques et sédimentologiques, un modèle d'érosion qui donne beaucoup plus d'importance à la valeur des débits de pointe de vidange du lac, qu'à la question des niveaux de base. De ce point de vue, la modification de la gestion des niveaux vers 1985, qui augmente encore ces vitesses de pointe (figure 8), peut être considérée comme un facteur aggravant (MERCIER \& SANCHEZ, 2010a ; 2010b). La première crise érosive documentée a duré grossièrement un demi-siècle et s'est traduite par un curage complet du lac et la disparition de presque tous les marais riverains. La seconde a été un peu plus courte et a représenté un curage moins sévère. Il est possible, si rien n'est fait, et vu les vitesses de vidange atteintes actuellement, que la crise actuelle puisse être la plus sévère des trois. Le risque est évidemment la destruction de la biodiversité qui justifie les mesures de protection et qui résulte de la faible profondeur du lac et de la présence des marais riverains.

\subsection{La question de la qualité écologique du milieu et de la turbidité}

Il apparaît que la période où le lac a abrité le maximum de biodiversité (LLOYD, 1897 ; GADECEAU, 1909 ; MARION \& MARION, 1976 ; DUPONT, 2003) correspond globalement à la première moitié du $\mathrm{XX}^{\mathrm{ème}}$ siècle. C'est notamment la seule époque où l'eau était limpide et où d'importantes ceintures de macrophytes flottants et des herbiers sous-aquatiques remarquables prospéraient. Il y a un intérêt évident à s’intéresser aux modifications des facteurs abiotiques (ou autres) qui sont intervenues en même temps que la dégradation du milieu.

MARION \& MARION (1976), MARION (1992) et MARION (1999) ont donné beaucoup d'importance à l'envasement du lac et, à la dégradation de la qualité chimique des eaux venant du bassin versant, particulièrement en ce qui concerne les nutriments. En fait, la dynamique sédimentaire, nous l'avons vu, a été mal appréhendée (voir supra et MERCIER \& SANCHEZ, 2010a). Quant à la dégradation chimique, elle est indiscutable à l'échelle du demi-siècle (DUPONT, 2003); néanmoins, en ce qui concerne les nutriments, la situation semble s’être améliorée depuis quelques décennies. C'est au moins ce que l'on constate à l'échelle du pays : diminution puis interdiction des lessives au phosphate, et diminution de 70\% des phosphates par l'agriculture entre 
1975 et 2008 (DEGRON \& MARGONTIER, 2010) et, par voie de conséquence, diminution de 50\% des phosphates dans les cours d'eau entre 1998 et 2007 (DUBOIT, 2009 ). Dans le bassin de Grand-Lieu, la tendance n'est pas connue, mais une amélioration de la qualité chimique est hautement probable dans la mesure où, depuis 1995, ce bassin a bénéficié d'efforts spécifiques pour améliorer la maîtrise des effluents (SAGE Logne, Boulogne, Ognon et lac de Grand-Lieu). Pourtant la prolifération du plancton, et donc la turbidité de l'eau, a continué, et a même augmenté.

Ces considérations, et le fait que les eaux du lac étaient turbides à une époque où, a priori, les apports en nutriments du bassin versant étaient faibles (XVIII et XIX ${ }^{\text {ème }}$ siècles), nous conduisent à supposer qu'un autre paramètre contrôle la prolifération du plancton. Le fait que la période, dans l'histoire du lac, où l'eau était la plus limpide est précisément comprise entre une baisse importante du niveau d'eau (canal de la Martinière), et une remontée de ce niveau (écluse de Bouaye) suggère que le niveau d'eau constitue ce paramètre de contrôle.

On sait, qu’à quantité de nutriments égale, les lacs abritant une végétation aquatique présentent une bien meilleure transparence (JEPPESEN et al., 1990 ; 1997); cela résulte d'une interaction complexe entre les macrophytes et les microphytes qui inclut la simple compétition (STEPHEN et al., 1998 ; ROIJACKERS et al., 2004) et des stratégies d'agression chimique (WIUM-ANDERSEN et al., 1982 ; GROSS et al.,. 2003 ; BURKS et al., 2006)

On sait également que les populations de macrophytes flottants sont très sensibles à de faibles fluctuations du niveau d'eau (BLINDOW et al., 1993; GACIA \& BALLESTEROS, 1996, PAILLISSON \& MARION, 2006). On conçoit donc que la remontée du niveau d'étiage, au début des années 1960, ait pu déclencher et entretenir la chaîne d'événements suivante : (i) régression des ceintures de macrophytes flottants (DUPONT, 2003), (ii) augmentation de la biomasse de microphytes et donc de la turbidité, (iii) disparition des herbiers aquatiques. Fort malheureusement, en 1995, une mauvaise appréhension du rôle des macrophytes, accusés du prétendu envasement rapide du lac, a conduit à la décision d'augmenter encore une fois les niveaux estivaux (MARION, 1992 ; 1999 ; PAILLISSON \& MARION, 2005 ; 2006) et à aggraver la situation.

\section{Conséquences pour la gestion hydraulique du lac de Grand-Lieu}

La gestion actuelle des niveaux pose donc un problème par rapport à des objectifs de conservation de la biodiversité du lac de Grand-Lieu; elle favorise, entre autres, l'érosion du fond et des berges, l'appauvrissement des ceintures de macrophytes et l'augmentation des blooms algaires. Il convient donc de proposer des améliorations tout en gardant à l'esprit qu'il serait illusoire de fixer comme objectif à cette démarche, la restauration du lac dans son état du début du $\mathrm{XX}^{\text {ème }}$ siècle. En effet, en dehors des 
leviers sur lesquels on peut théoriquement jouer : la gestion hydraulique et, dans une moindre mesure, le flux de nutriments; de nombreux paramètres, potentiellement déterminants, ont évolué sans qu'un retour aux conditions initiales ne semble possible :

(i) modification quantitative et qualitative de la pression de la pêche professionnelle. Au début du siècle, cette pêche était pratiquée par plus de 120 professionnels et concernait essentiellement les cyprinidés, alors qu'actuellement (SNPN 2009) elle concerne 7 personnes qui opèrent, pour $90 \%$ de la biomasse capturée, aux dépens de trois espèces "nobles" : l'Anguille (Anguilla anguilla), le Brochet (Esox lucius) et le Sandre (Sander lucioperca). On sait que les modifications du peuplement ichtyologique peuvent engendrer des modifications écologiques majeures, y compris dans l'équilibre macrophytes/microphytes (SCHEFFER, 1998)

(ii) installation massive d'espèces allochtones. Certaines de ces espèces ont un impact très significatif sur le milieu (ragondin (Myocastor coypus), rat musqué (Ondatra zibethicus), écrevisse de Louisiane (Procambarus clarkii)....

(iii) abandon du fauchage des joncs. Les joncs, essentiellement le phragmite (Phragmites australis), étaient exploités dans le lac pour alimenter une usine pratiquant le tressage et qui a fermé en 1949 (ANDRE et al., 2000). L'exportation de la matière organique hors du milieu lacustre, contribue à la maîtrise de son niveau trophique et donc à limiter le risque de bloom algaire.

(iv) modification des équipements hydrauliques. Le recalibrage de l'Acheneau qui multiplie la capacité de vidange du lac et la création de l'écluse de Bouaye qui permet le contrôle du niveau, constituent des facteurs d'artificialisation du milieu, mais inversement offrent un levier de contrôle sur un facteur abiotique déterminant.

Depuis 1995, la doctrine qui constitue la base de la gestion des niveaux printaniers et estivaux du lac repose sur les trois postulats suivants (MARION, 1999 ; PAILLISSON \& MARION, 2005) :

- le lac subit un envasement annuel pluri-centimétrique ;

- cet envasement est essentiellement relatif à la matière organique autochtone et spécialement aux macrophytes flottants ;

- le meilleur moyen pour lutter contre ces macrophytes consiste à maintenir des niveaux hauts au printemps pour les asphyxier (PAILLISSON \& MARION, 2005 ; 2006).

Cette conception s'est traduite par la promulgation d'un arrêté ministériel qui a remonté fortement les cotes. Cet arrêté fut aménagé en 1996 et 2004 suite à des conflits avec les riverains (figure 6). Cette politique de niveaux estivaux hauts amplifie de facto un mouvement commencé dès la création de l'écluse de Bouaye, au début des années 1960 (figure 6).

Nous avons établi que le premier postulat est inexact : le lac ne s'envase pas. De ce fait, il n’y a aucun intérêt à limiter les macrophytes flottants. Par ailleurs, la chasse aux 
canards n'est plus un objectif de gestion du lac qui est intégralement classé en réserves nationale ou régionale. Enfin, nous l'avons vu, il existe des indices très forts qui suggèrent qu'une baisse significative des niveaux printaniers et estivaux favoriserait la biodiversité et l'intérêt écologique du milieu grâce (i) à la reconquête de l'espace lacustre par les macrophytes flottants et les herbiers aquatiques et (ii) à la compétition que ces derniers exercent sur les microphytes. Cette reconquête de l'espace lacustre par la végétation fixée, aurait, de plus, une influence directe sur la vitesse des courants et la remise en suspension des sédiments et donc au final sur l'érosion (DIETER, 1990 ; JAMES \& BARKO, 1994 ; MADSEN et al., 2001 ; JAMES et al., 2004).

La baisse des niveaux plus tôt au printemps semble donc souhaitable. Mais, d'un autre côté, MERCIER \& SANCHEZ (2010a ; 2010b) ont insisté sur le fait qu'à la sortie de l'hiver, des baisses de niveau trop rapides, notamment lors d'épisodes de fort vent, à l'occasion desquels les sédiments sont remis en suspension, favorisent l'érosion lacustre. Enfin, REEBER (2000) pour l'avifaune et DUPONT (2003) pour la végétation ont souligné la nécessité de réintroduire, au printemps et en été, une variabilité interannuelle des niveaux qui a disparu à partir de 1962, pour les étiages précoces, et de 1985, pour les crues tardives (figure 6).

L'ensemble de ces contraintes, qui ne sont pas totalement compatibles en première analyse, conduisent à suggérer la mise en place d'une gestion hydraulique complexe qui tienne compte de facteurs variés comme la force du vent, la teneur en MES minérale des eaux, la dynamique sédimentaire...

Vu les conflits antérieurs qui se sont focalisés sur les questions d'hydraulique, et la lenteur des évolutions naturelles, une démarche d'amélioration de la gestion par tâtonnement ne paraît ni souhaitable, ni même réaliste. La réalisation d'un modèle numérique de simulation de l'évolution hydro-sédimentaire du lac est donc souhaitable. Ce projet, qui est en cours d'élaboration, se propose de tester divers scenarii caractérisés par :

(i) des modifications éventuelles des exécutoires du lac (suppression ou modification du canal Guerlain par exemple, MERCIER \& SANCHEZ, 2010a)

(ii) un pilotage des débits et non plus des niveaux. Ce type de gestion irait dans le sens d'une baisse moyenne des niveaux printaniers et estivaux, d'une limitation de l'érosion, mais également dans le sens d'une variabilité interannuelle. Ainsi, un haut niveau hivernal se traduirait par une vidange longue, et donc des niveaux printaniers hauts et un hiver sec, par un étiage précoce et des niveaux estivaux plus bas. Une telle variabilité interannuelle réintroduirait un peu de "naturalité" dans l'hydraulique du lac. 


\section{Bibliographie}

ANDRE J., COULON M.J., NAUD C. (2000). A Grand-Lieu, un village de pêcheurs. Passay se raconte. Siloë, $316 \mathrm{p}$.

ANONYME (commission spéciale) (1851). Annuaire des eaux de la France pour 1851. Imprimerie nationale, Paris.

ANONYME (entre 1894 et 1899). Le dessèchement du Lac de Grand-Lieu Le lac avant et après. (ADLA $1781 \mathrm{~S} 4)$.

ANONYME (1999). Système d'évaluation de la qualité des cours d'eau, principes généraux. Agences de l'eau, les études des agences de l'eau n 64, 21 p.

BARON L. (1888). La Loire. Laurens, Paris.

BARRON. L. (1899). Le nouveau voyage en France. Mame, Tours.

BLINDOW I., ANDERSSON G., HARGEBY A., JOHANSSON S. (1993). Long-term pattern of alternative stable states in two shallow eutrophic lakes. Fresh-water Biol., Vol. 30, pp 159-167. doi:10.1111/j.1365-2427.1993.tb00796.x

BORET P. (2005). Bathymétrie de la zone centrale du lac de Grand-Lieu. Document non publié, SNPN, Paris.

BORET P., REEBER S. (2008). Suivi spatial de la zone centrale du lac de Grand Lieu entre 1993 et 2007. SNPN, Paris.

BOURRIGAUD R. (2007). La genèse des institutions locales de gestion de l'eau au XVIIIe siècle, p. 145-198. In "Gouvernance et partage de l'eau. Le bassin versant de Grand-Lieu", Presses Univ. Rennes, 204 p.

BURKS R.L., MULDERIJ G., GROSS E., JONES J.I., JACOBSEN L., JEPPESEN E., VAN DONK E. (2006). Center stage: the crucial role of macrophytes in regulating trophic interactions in shallow lake wetlands, p. 37-59. In R. Bob- bink, B. Beltman, J. T. A. Verhoeven, and D. F. Whigham, editors. "Wetlands: Functioning, Biodiversity Conservation, and Restoration". Springer-Verlag, Berlin Heidelberg.

BRUNAUD D. (2007). Etangs pelliculaires et réserves naturelles : principes de fonctionnement et modalités de classement. Univ. Limoges, thèse de doctorat en géographie, décembre 2007, 582 p.

COMMISSARIAT AU PLAN (1994). Les zones humides, rapport de l'instance d'évaluation . La Documentation française.

CONSTANTIN P., RODET P. (1892). Manuel de thérapeutique clinique, les eaux de table. Asselin et Houzeau edt., Paris.

DEGRON R., MARGONTIER S. (2010). L'environnement en France. Service de l'observation et des statistiques, Commissariat général au développement durable, $150 \mathrm{p}$.

DELEBECQUE A. (1898). Les lacs français. Chamerot \& Renouard, Paris, 436 p. DE MARSILY G. (2006). Les eaux continentales. Academie des Sciences, 319 p. 
Trois siècles d'histoire hydro-sédimentaire et écologique du lac de Grand-Lieu, conséquences pour la gestion hydraulique : 6.23

DERIC G. (1847). Histoire ecclésiastique de Bretagne. t 1, Prud'Homme edt, Saint Brieuc.

DIETER C.D. (1990). The importance of emergent vegetation in reducing sediment resuspension in wetlands. J. Freshwat. Ecol., Vol. 5, pp 467-473. doi:10.1080/02705060.1990.9665263

DUBOIT A. (2009). Evolution de la qualité des cours d'eau : volet macropolluants. Commissariat général au développement durable, $50 \mathrm{p}$.

DUGAST-MATIFEUX C. (1879). Nantes ancien et le pays nantais. Morel.

DUPONT P. (2003). L'évolution de la flore et de la végétation du lac de Grand-Lieu (Loire Atlantique) et de ses ceintures : situation actuelles, problèmes de conservation et de gestion. Bulletin de la Société Botanique du Centre-Ouest, Vol. 34, pp 3-64.

FREOR P. (1979). Le lac de Grand-Lieu, les binet de Jasson, le cheval Mallet. Edition du Pays de Retz, 93 p.

GACIA E., BALLESTEROS E. (1996). The effect of increased water level on Isoetes lacustris L. in Lake Baciver, Spain. J. Aquat. Plant Manage, Vol. 34, pp 57-59.

GADECEAU E. (1909). Le lac de Grand-Lieu. Monographie phytogéographique. Nantes, 155 p.

GIRAULT DE SAINT-FARGEAU (1829a). Dictionnaire géographique des communes de France. Lycée armoricain, V 13, 400 p.

GIRAULT DE SAINT-FARGEAU (1829b). Histoire nationale et dictionnaire géographique de toutes les communes de la Loire-Inférieure. Baudoin edt, Paris.

GROSS E.M., ERHARD D., IVANYI E. (2003). Allelopathic activity of Ceratophyllum demersum L. and Najas marina ssp. intermedia (Wolfgang) Casper. Hydrobiologia Vol. 506/509, pp 583-589.

GUICHARD F. (1887). Notice sur le lac de Grand-Lieu. $8^{\text {ème }}$ Congrès national des sociétés françaises de géographie, pp 171-183.

GUILLOU J.-J., MERCIER E. SANCHEZ M. (2008). Influence des aménagements hydrauliques sur l'évolution des niveaux d'eau dans le lac de Grand-lieu depuis le XVIIIe siècle. Bull. Soc. Historiens Pays de Retz, Vol. 27, pp 21-30.

HERY-VIOLETTE J.M., ARCHAMBAULT P.J. (1859). Dictionnaire des analyses chimiques. $t$ 1, Lacroy et Baudy, Paris.

JAMES W.F., BARKO, J.W. (1994). Macrophyte influences on sediment resuspension and export in a shallow impoundment. Lake and Reservoir Management, Vol. 10, pp 95-102. doi:10.1080/07438149409354180

JAMES W.F., BEST E.P., BARKO J.W. (2004). Sediment resuspension and light attenuation in peoria lake : can macrophyte improve water quality in this shallow system ? Hydrobiologia, Vol. 515, pp 193-201. doi:10.1023/B:HYDR.0000027328.00153.b2

JEPPESEN E., SØNDERGAARD M., JENSEN J.P., KANSTRUP E., PETERSEN B. (1997). Macrophytes and turbidity in brackish lakes with special emphasis on top-down 
control. In: Jeppesen E, Søndergaard Ma, Søndergaard Mo, \& Christoffersen K, editors. "The structuring role of submerged macrophytes in lakes". Heidelberg: Springer, pp 369-77.

JEPPESEN E., JENSEN J.P., KRISTENSEN P., SØNDERGAARD M., MORTENSEN E., SORTKJÆR O., OLRIK K. (1990). Fish manipulation as a lake restoration tool in shallow, eutrophic, temperate lakes 2: threshold levels, long-term stability and conclusions. Hydrobiologia, Vol. 200/201, pp 219-27. doi:10.1007/BF02530341

JIGOREL A. (1992). Etude sédimentologique du lac de Grand-Lieu. INSA Rennes, 83 p.

LAVERAN (1898). Traité du paludisme. Masson, Paris, 492 p.

LEFORT J. (1859). Chimie Hydrologique. Masson, Paris.

LESCADIEU A., LAURANT A. (1836). Histoire de la ville de Nantes, suivie de l'histoire des guerres de Vendée. t. I, Laurant, Nantes, 413 p.

LLOYD J. (1844). Flore de la Loire Inférieure. Prosper Sebire, Nantes, 334 p.

LLOYD J. (1854). Flore de l'Ouest de la France. $1^{\text {ère }}$ édt., Forest ainé, Nantes, 576 p.

LLOYD J. (1869). Flore de l'Ouest de la France. $2^{\text {ème }}$ édt., Veloppé, Nantes 644 p.

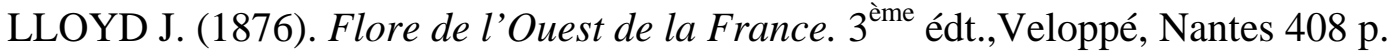

LLOYD J. (1886). Flore de l'Ouest de la France. 4 ème édt., Veloppé, Nantes 455 p.

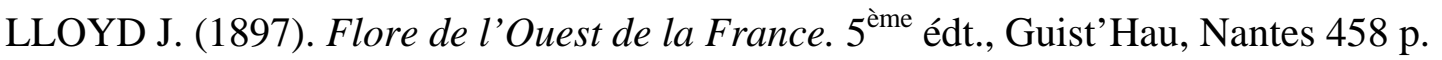

MADSEN J.D., CHAMBERS P.A., JAMES W.F., KOCH, E.W., WESTLAKE D.F. (2001). The interaction between water movement, sediment dynamics and submersed macrophytes. Hydrobiologia, Vol. 444, pp 71-84. doi:10.1023/A:1017520800568

MAITRE L. (1912). Le Lac de Grandlieu et ses affluents. Res Universis édition en facsimilé de 1993, $232 \mathrm{p}$.

MAITRE L. (1912-1915). Géographie physique du Lac de Grandlieu et de ses affluents. Annales de Bretagne, (28) 63-75 \& 229-238, (29) 138-151 \& 679-707, (30) 82-106.

MANET F.G.P.B. (1834). Histoire de la petite Bretagne ou Bretagne armorique. t. II, Caruel, Saint Malo.

MARION L. (1992). Lac de Grandlieu: causes de l'envasement et mesures de sauvetage. Rapport de Synthèse. Université de Rennes, 71 p.

MARION L. (1999). Le lac de Grand-Lieu, un joyau tropical à préserver. Réserve naturelle du lac de Grand-Lieu, SNPN, 64 p.

MARION L., MARION P. (1976). Contribution à l'étude écologique du lac de GrandLieu. SSNOF, suppl. 611 p., Nantes.

MERCIER E., SANCHEZ M. (2010a). Essai de bilan sédimentation/érosion du lac de Grand-lieu (Loire-Atlantique) : conséquences pour la gestion du milieu naturel. Rev. Écol. (Terre Vie), Vol. 65, pp 193-208.

MERCIER E., SANCHEZ M. (2010b). Influence de la gestion hydraulique sur la 
Trois siècles d'histoire hydro-sédimentaire et écologique du lac de Grand-Lieu, conséquences pour la gestion hydraulique : 6.25

dégradation des milieux du Lac de Grand-Lieu : éléments de discussion. XI ${ }^{\mathrm{èmes}}$ Journées Nationales Génie Côtier - Génie Civil, Les Sables d’Olonne, 22-25 juin 2010, pp 361-368. doi:10.5150/jngcgc.2010.043-M

MIGNIOT C., LE HIR P. (1994). Estuaire de la Loire, t 1, Hydrosédimentaire. APEEL, 83 p.

MILlE X. (1859). Dessèchement du Lac de Grand-Lieu, Avant projet, rapport de l'ingénieur en chef. Manuscrit, 16 p., (ADLA, 1781 S3).

NOTHOMMES G. (2005). Evolution de l'agriculture au XIXè siècle et culture céréalière autour du lac de Grand-Lieu. Bulletin de la Société des Historiens du Pays de Retz, Hors Série $n^{\circ} 1$; pp 29-36.

PAILLISSON J.M., MARION L. (2005). Productivité des macrophytes flottants du lac de Grand-Lieu (saison 2004). Effets de paramètres environnementaux. DIREN et Université de Rennes 1.

PAILLISSON J.M., MARION L. (2006). Can small water level fluctuations affect the biomass of Nymphaea alba in large lakes ? Aquatic Botany, Vol. 84, pp 259-266. doi:10.1016/j.aquabot.2005.10.004

PELIEU J.B. (1852). Les anciennes mesures du département de la Loire inférieure comparées aux nouvelles et réciproquement. Forest, Nantes.

PELTIER D. (2004). Proposition d'une méthodologie pour la localisation de la zone humide du lac de Grand Lieu conformément au S.A.G.E Logne, Boulogne, Ognon et Grand Lieu. CLE Grand-lieu.

PIEGAY H., PAUTOU G., RUFFINONI C. (2003). Les forêts riveraines des cours d'eau: écologie, fonctions et gestion. Institut pour le développement forestier, $460 \mathrm{p}$.

PIERRELEE D., LAMPRIER A. (2004). Grand-Lieu, lac et marais : mémoire des paysages entre Loire et baie. Siloë, 293 p.

PIGNANIOL DE LA FORCE (1754). Description de la France. t 8, Poiron, Paris.

PINSON F.J. (1888). Plan cadastral du canton de Saint Philibert de Grand-Lieu. (ADLA, 1 Fi Loire-Atlantique 19/27).

PITRE AUBINAIS (1855). Quelques réflexions sur le fièvres périodiques pernicieuses. Journal de la section de Médecine de la Société Académique de département de la Loire inférieure, Vol. 31, pp 64-82.

RAMADE F. (1984). Eléments d'écologie, écologie fondamentale. Me Graw Hill, Paris, $397 \mathrm{p}$.

REEBER S. (2000). Impact du relèvement du niveau d'eau du lac de Grand-Lieu sur l'avifaune. SNPN, Paris.

RIEFFEL J. (1834). Du défrichement des landes en Bretagne. Annales agricoles de Roville, Vol. 9, pp 331-362. 
ROIJACKERS R., SZABO S., SCHEFFER M. (2004). Experimental analysis of the competition between algae and duckweed. Archiv für Hydrobiologie, Vol. 160(3), pp 401-412. doi:10.1127/0003-9136/2004/0160-0401

SCHEFFER M. (1998). The ecology of shallow lakes. Chapman \& Hall, London, 357 p. SNPN (2009). Plan de gestion de la Réserve Naturelle Nationale du lac de Grand-Lieu, 2009-2013. SNPN, 319 p.

STEPHEN D., MOSS B., PHILLIPS G. (1998). The relative importance of top-down and bottom-up control of phytoplankton in a shallow macrophyte-dominated lake. Freshwater Biology, Vol. 39, pp 699-713. doi:10.1046/j.1365-2427.1998.00321.x STRUILLOU J.F., MENANTEAU L., CHADENAS C., POURINET L. (2006). Protection du patrimoine naturel et développement durable dans l'estuaire de la Loire. Le cas du lac de Grand-Lieu. Colloque International Interactions Nature-Société : analyses et modèles, La Baule (Loire-Atlantique - France) 3-6 mai 2006, 7 p.

TALUREAU R. (1965). Marais de l'Ouest. Les grands aménagements ruraux. Ministère de l'agriculture, 180 p.

VALLES F. (1848). Projet de desséchement et d'irrigation du lac de Grand-Lieu. Annales des Ponts et Chaussées, pp 158-251.

VAYSSE DE VILLERS M. (1831). Itinéraire descriptif de la France, Région SudOuest. Renouard, Paris.

VEZIER-VAUTHIER A. (1992). Le canal maritime de la Basse Loire hier et aujourd'hui. Bull. Soc. Etudes et recherches historiques du Pays de Retz, Vol. 12, pp 10-25.

VOYER ARGENSON M.A.R. DE (1783). Mélange tiré d'une grande bibliothèque de lecture des livres François. t 7, Moutard, Paris.

WIUM-ANDERSEN S., ANTHONI U., CHRISTOPHERSEN C., HOUEN G. (1982). Allelopathic effects on phytoplankton by substances isolated from aquatic macrophytes (Charales). Oikos, Vol. 39, pp 187-190. doi:10.2307/3544484 